\title{
A case to identify the factors causing a disaster with risk reception
}

\author{
Rundong Wang ${ }^{1,2,3}$, Chongfu Huang ${ }^{1,2,3, *}$, Yiran Yin ${ }^{1,2,3}$ \\ ${ }^{1}$ Key Laboratory of Environmental Change and Natural Disaster, Ministry of Education, Beijing Normal University, Beijing 100875, China \\ ${ }^{2}$ State Key Laboratory of Earth Surface Processes and Resources Ecology (Beijing Normal University), Beijing 100875, China \\ ${ }^{3}$ Academy of Disaster Reduction and Emergency Management, Faculty of Geographical Science, Beijing Normal University, Beijing \\ 100875, China \\ 710744138@qq.com,hcongfu@bnu.edu.cn
}

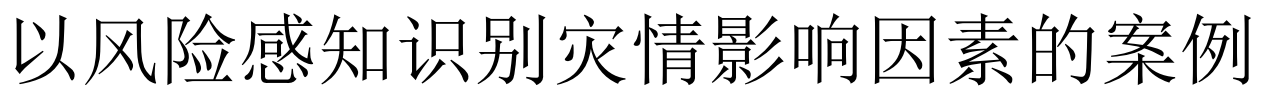

\author{
王润东 $1,2,3$, 黄崇福 $1,2,3, *$, 尹怡然 $1,2,3$ \\ 1 北京师范大学, 环境演变与自然灾害教育部重点实验室, 北京100875, 中国 \\ 2 北京师范大学，地表过程与资源生态国家重点实验室，北京 100875 , 中国 \\ 3 北京师范大学, 地理科学学部, 减灾与应急管理研究院, 北京 100875 , 中国 \\ 710744138@qq.com, hcongfu@bnu.edu.cn
}

\begin{abstract}
There are many factors affecting the disaster situation, and weights of various factors are different. Theoretical analysis results are only applicable to disasters and regions that meet theoretical assumptions. Based on the knowledge of some people about local earthquake disasters and flood disasters in Santai County, this paper proposes a method to identify the factors affecting disasters by risk perception. In the case, risk perception is reflected in the score of affecting factors' importance. The study found that the background of the respondents is closely related to their risk perception results. The factors affecting the disaster can be summarized as risk source factors, emergency ability factors and vulnerability factors. This study can provide a reference for the weights calculation in relative exposure.
\end{abstract}

Keywords - relative exposure, risk perception, disaster factors, correlation analysis, factor analysis

摘要 一 影响灾情的因素很多, 且各种因素的权重不同。 理论分析结果, 仅适用于满足理论假设的灾种和区域。本文 根据三台县部分民众对当地地震灾害和洪水灾害的认知, 提 出了以风险感知识别灾情影响因素的方法。案例中, 风险感 知体现在对影响因素重要性的评分上。研究发现: 被调查者 的背景与其风险感知结果密切相关。灾情影响因素可以概括 为风险源因子、应急能力因子和脆弱性因子。这一研究可以 为相对暴露度中权重计算提供参考。

关键词一相对暴露度, 风险感知, 灾情影响因素, 相关 性分析，因子分析

\section{I. 引言}

亲身经历过灾害的人对灾害会有更加清晰的认知。近 年来, 伴随着社会经济的高速发展、全球气候变化, 自 然灾害异常增加, 同时灾害损失强度明显加剧。灾害管 理不但要重视灾后的反应措施, 更要重视灾前的防御工 作，从灾后“响应文化”向灾前“预防文化”的转变具有重 要意义。所以, 就必须重视公众对于灾害的风险感知。

苏桂武等[1]以汶川大地震重灾区之一的四川省德阳 市为例, 借助问卷调查和现场访谈, 分析了灾区民众认
知与响应地震灾害的一些整体特点。谢晓非等[2]主要从 心理学的角度出发, 着重研究了个体差异、灾害水平、 通讯的影响、自愿承担风险的程度、风险的性质等影响 个体风险认知的因素, 并且研究风险知识的来源, 个体 的环境风险知识很少是直接经验, 绝大部分来自于信息 的传播与沟通。

自然灾害领域尚未对自然灾害风险暴露有一个明确的 定义，黄崇福[3]将自然灾害风险暴露于定义为“自然灾 害风险暴露是生命和人类社会财产的未来是否容易遭受 自然事件或力量打击的一种状态”，并且提出了更易度量 的相对暴露, 给出定义“设 $A, B$ 是两个面对同一风险源 的风险承受体。如果此风险源暴发时, $A$ 比 $B$ 更容易遭 受打击, 称 $A$ 的相对暴露比 $B$ 高”。

因子分析法 $[4,5]$ 是指从研究指标相关矩阵内部的依赖 关系出发, 把一些信息重叠、具有错综复杂关系的变量 归结为少数几个不相关的综合因子的一种多元统计分析 方法。基本思想是：根据相关性大小把变量分组，使得 同组内的变量之间相关性较高, 但不同组的变量不相关 或相关性较低, 每组变量代表一个基本结构一一即公共 因子，在损失很少信息的前提下达到降维的目的。李小 胜等 $[6,7]$ 对常见的因子分析进行了总结, 对其中的错误 进行了改正, 从原理的角度剖析了因子分析。

\section{II. 调研过程}

\section{A. 问卷设计}

灾害认知是个体对自然灾害客观风险的主观感受与认 识, 而这些主观感受会受到性别、年龄、职业等因素的 影响 [8]。灾害认知的研究主要基于认知心理学和行为地 理学。人脑接受、分析处理信息的能力体现为认知水平 和意识, 进而会影响到内在行为倾向和外在调试行为 等。通过调研，本文总结出了 13 个影响地震灾害损失的 因素（后续分析简称为 $\mathrm{dz} 01-\mathrm{dz} 13$ ）和 15 个影响洪水灾 害损失的因素（后续分析简称为 hs01-hs15），并据此设

*通讯作者：黄崇福，教授, Email: hcongfu@bnu.edu.cn 
计了调查问卷《地震、洪水灾情影响因素调查问卷》。 问卷涉及了被调查者的性别、出生年份、职业与居住 地, 要求被调查者对每一项进行重要性评级, 分为极 低、很低、较低、较高、很高、极高 6 个级别。

表 1 地震、洪水灾情影响因素

\begin{tabular}{ll}
\hline 地震灾情影响因素 & 洪水灾情影响因素 \\
\hline 震级 $d z 01$ & 降水量 $h s_{01}$ \\
震中距 $d z 02$ & 淹没范围 $h s_{02}$ \\
震源深度 $d z 03$ & 淹没深度 $h s_{03}$ \\
地质构造特点 $d z 04$ & 淹没时长 $h s_{04}$ \\
房屋结构 $d z 05$ & 最大径流量 $h s_{05}$ \\
经济发达程度 $d z 06$ & 农田分布情况 $h s_{06}$ \\
人口密度 $d z 07$ & 房屋结构 $h s_{07}$ \\
居民自救互救能力 $d z 08$ & 经济发达程度 $h s_{08}$ \\
地震发生时间 $d z 09$ & 人口密度 $h s_{09}$ \\
基础措施的完备程度 $d z 10$ & 居民自救互救能力 $h s_{10}$ \\
救援力度 $d z 11$ & 洪水发生时间 $h s_{11}$ \\
救援速度 $d z 12$ & 基础设施完备程度 $h s_{12}$ \\
是否引起次其他生灾害 $d z 13$ & 救援力度 $h s_{13}$ \\
& 救援速度 $h s_{14}$ \\
& 是否引起次其他生灾害 $h s_{15}$ \\
\hline
\end{tabular}

\section{B. 研究区简介}

2019 年 6 月, 调研工作在在四川省绵阳市三台县展 开。该县位于四川盆地中偏西北部的, 属于川中丘陵地 区, 地势北高南低, 是个农业大县。其境内有河流 46 条, 并包括了 4 条大江, 区域洪水风险较高。“5.12”汶 川地震及后来数次小地震对该地区也有一定的影响, 造 成了部分居民房屋的垮塌, 当地村民主要经济来源麦冬 减产甚至绝收从而严重阻碍了区域农业的发展。三台县 经常受到地震、洪涝综合风险的影响。

\section{C. 调研过程}

调研采用线上 (微信、网页) 和线下 (座谈、入户一 对一) 两种方式结合发放调查问卷。线下主要在新鲁 镇、花园镇、永明镇、老马乡、里程乡和争胜镇 6 个乡 镇及所辖村落发放。调研回收了 24 个乡镇的数据, 包括 三台县城、北坝镇、东塔镇、富顺镇、古井镇等 24 个乡 镇的问卷, 其中以里程乡、刘营镇、争胜镇三个乡镇的 问卷居多。实际发放问卷 509 份, 回收有效问卷为 479 份, 可满足研究需要。经过统计, 得到被调查样本的基 本属性如表 2 所示。

表 2 调查样本基本属性

\begin{tabular}{|c|c|c|c|c|c|c|c|c|}
\hline \multirow{2}{*}{ 类别 } & \multicolumn{2}{|c|}{ 性别 } & \multicolumn{6}{|c|}{ 职业 } \\
\hline & 男 & 女 & 务农 & 公务员 & & 工人 & 学生 & 其他 \\
\hline 人数/人 & 226 & 253 & 29 & 17 & & 13 & 345 & 75 \\
\hline 百分比 $/ \%$ & 47.2 & 52.8 & 6.1 & 3.5 & & 2.7 & 72 & 15.7 \\
\hline \multirow[b]{2}{*}{ 类别 } & \multicolumn{4}{|c|}{ 户籍 } & \multicolumn{4}{|c|}{ 年龄段 } \\
\hline & 里程 & 刘营 & 争胜 & 其他 & 1-10 & $\begin{array}{l}11- \\
18\end{array}$ & $\begin{array}{l}\text { 19- } \\
40\end{array}$ & $>40$ \\
\hline 人数/人 & 206 & 41 & 162 & 70 & 184 & 170 & 78 & 47 \\
\hline 百分比 $/ \%$ & 43 & 8.6 & 33.8 & 14.6 & 38.4 & 35.5 & 16.3 & 9.8 \\
\hline
\end{tabular}

职业被分为了务农、公务员、工人、学生与其他职业 进行分类统计, 由于调研过程与教育部门对接, 导致学 生所占比例高, 占到了 $72 \%$ 。户籍被分为了里程乡、刘
营镇、争胜镇与其他乡镇进行统计。由于学生在受访民 众中所占比例大, 老年人较少, 所以将年龄段分为了 110 岁、11-18 岁、19-40 岁和 40 岁以上进行统计。

\section{III. 数据分析}

\section{A. 定类变量与定序变量的量化处理}

在本问卷所涉及的各个指标中, 存在着定类变量与定 序变量。定类变量的类与类互斥, 研究对象均有所属, 具有 $=$ 和 $\neq$ 的数学性质, 只有类别之分, 没有大小程度 之分 $[9,10]$, 如本问卷中的性别和职业。而定序变量是区 别同一类别个案中等级次序的变量, 是比定类变量层次 更高的变量, 除了具有定类变量所具有的 $=$ 和 $\neq$ 的数学 性质外, 还具有 $>$ 和 $<$ 的数学性质, 如本问卷中民众对 于“基础设施的完备程度”这一因素对于地震灾情影响的 评级。

为了适应数据分析的需要, 首先应对定类和定序变量 进行量化处理。

借助虚拟变量来量化定类变量, 可用 $\mathrm{k}$ 个取值为 0 和 1 的虚拟变量分别代表各类的属性, 当问卷内容属于一 个虚拟变量所代表的类时, 这个虚拟变量就赋值为 1 , 否则为 0 。对二分定类变量, 用 1 个取值为 0 和 1 的虚 拟变量即可, 比如性别, 在本问卷中, 将男性赋值为 0 , 女性赋值为 1 , 即将性别这一定类变量转化为取值 0,1 的虚拟变量达到量化的效果。对于定类变量的类别是 2 个以上时, 用 $\mathrm{k}$ 个虚拟变量来表示, 如户籍分类, 在 本问卷处理过程中, 将调查对象的户籍分为了里程乡、 刘营镇、争胜镇与其他乡镇, 可将其转化为 4 个虚拟变 量（如表 3 所示）, 户籍通过虚拟变量的下角标来量 化。

表 3 户籍类别及其虚拟变量

\begin{tabular}{ccccc}
\hline 原变量类 & \multicolumn{4}{c}{ 虚拟变量 } \\
\cline { 2 - 5 } 别 & $\boldsymbol{X}_{\mathbf{0}}$ (其他) & $\boldsymbol{X}_{\mathbf{1}}$ (里程乡) & $\boldsymbol{X}_{\mathbf{2}}$ (刘营镇) & $\boldsymbol{X}_{\mathbf{3}}$ (争胜镇) \\
\hline 刘营镇 & 0 & 0 & 1 & 0 \\
争胜镇 & 0 & 0 & 0 & 1 \\
里程乡 & 0 & 1 & 0 & 0 \\
其他 & 1 & 0 & 0 & 0 \\
\hline
\end{tabular}

由于量表其自身的测量尺度就是一种定序尺度, 其测 量结果属于定序变量。可以根据其等级极低、很低、较 低、较高、很高、极高转化为 $1 、 2 、 3 、 4 、 5 、 6$, 这样 每一个等级就完成了量化, 定序变量之间可相互比较, 但不能进行精准的测量和运算。

\section{B. 整体分析}

我们整理了灾情影响因素的调查问卷结果, 并进行了 整体的分析（如图 1 所示）。在地震灾情的影响因素 中, 受访者们认为重要程度最大的是“救援速度”、“救援 力度”, 其次是“震级”、“震源深度”、“人口密度”、“房 屋结构”，而受访者认为“地质构造”、“震中距”等因素对 地震灾情影响较小, 其中, 尤以“发生时间”、“经济发达 程度”影响最小。受访者比较注重政府的应急能力, 而对 风险源和风险承受体关注较少。 


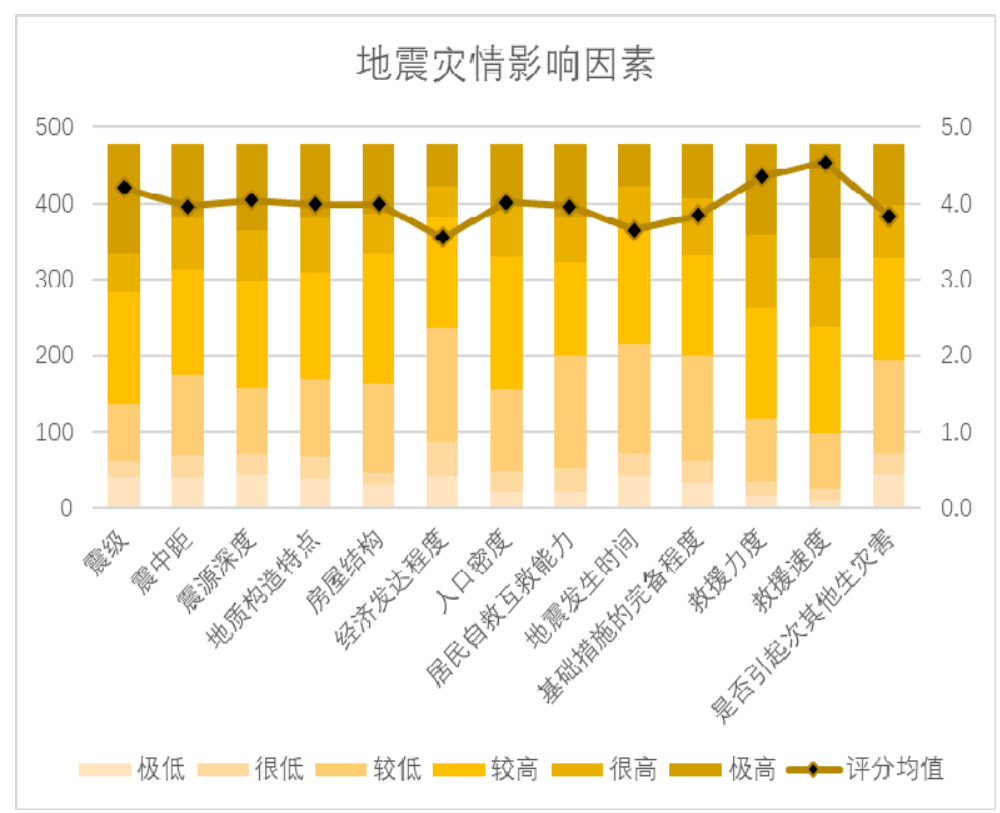

图 1 地震灾情影响因素整体分析

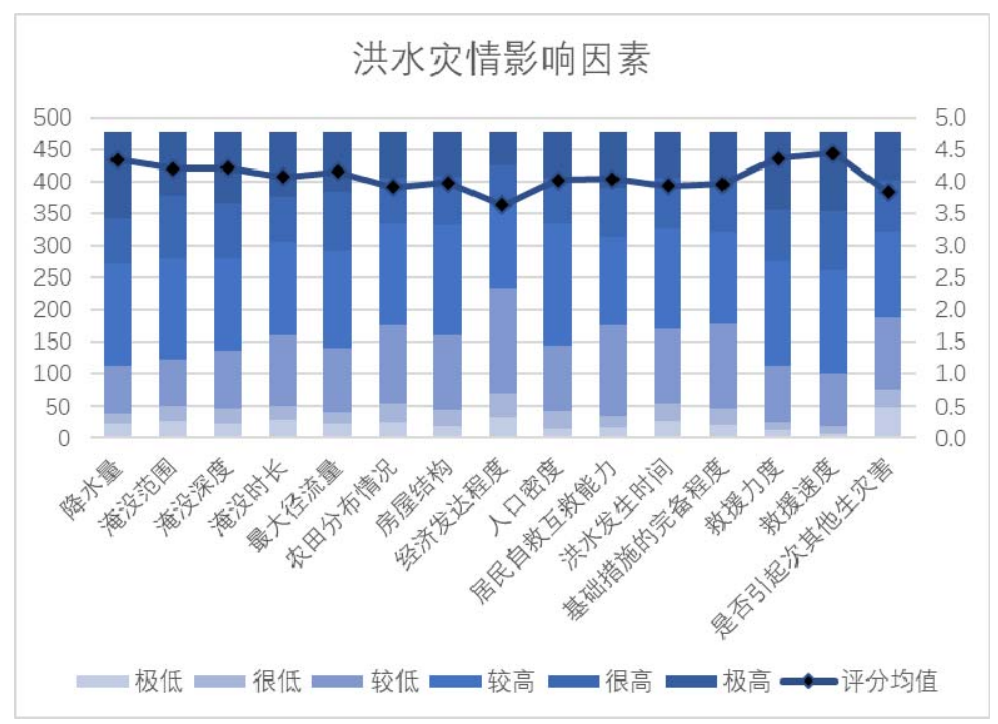

图 2 洪水灾情影响因素整体分析

而对于洪水灾情的影响因素中（如图 2 所示）, 受访 者们认为重要程度最大的是“救援速度”、“救援力度”, 其次是“降水量”、“淹没深度”、“淹没范围”、“最大径流 量”、“淹没时长”，而受访者认为“居民自救互救能力”等 因素对洪水灾情影响较小, 其中, 尤以 “经济发达程 度”、“农田分布情况”影响最小。受访者依然比较注重政 府的应急能力, 但与地震灾情影响因素不同的是, 对于 洪水灾情影响因素, 受访者们更注重风险源, 最后才是 风险承受体。

\section{C. 相关性分析}

影响受访者打分的因素有很多, 一般来说, 其自身的 性别, 户籍或是年龄都可能会影响受访者的判断。而调 查结果中多为定类变量和定序变量, 故可以采用基于交 叉表的频数和卡方检验来判断定类变量与定序变量的相
关性水平; 利用 Kendall 相关性分析来对定序变量之间进 行相关性分析。

以年龄段与震级为例, 使用统计软件 SPSS20.0 对调 研结果进行相关性分析, 震级*年龄段交叉制表如表 4 所 示, 可以直观地发现年龄段与震级存在一定的相关性。 并且卡方检验中 Pearson 卡方、似然比的检验概率分别为 0.009 与 $0.005(<0.05)$, 说明了震级评分与年龄段存在 显著相关。

分析表明, 受采访者的年龄段、户籍以及其职业是否 为学生均与部分指标存在相关性。尤其是学生在 “震 级”、“震中距”、“震源深度”、“次生灾害”等涉及专业术 语的指标中与非学生存在显著性差异, 可能是由于学生 不太懂专业术语的含义所致。 
表 4 震级*年龄段交叉表

\begin{tabular}{|c|c|c|c|c|c|}
\hline 震级 ${ }^{\text {年龄段 }}$ & $1-10$ & $10-18$ & $19-40$ & 40 以上 & 合计 \\
\hline 1 & 13 & 23 & 3 & 3 & 42 \\
\hline 2 & 10 & 6 & 4 & 0 & 20 \\
\hline 3 & 28 & 31 & 11 & 5 & 75 \\
\hline 4 & 68 & 47 & 24 & 7 & 146 \\
\hline 5 & 15 & 20 & 9 & 8 & 52 \\
\hline 6 & 50 & 43 & 27 & 24 & 144 \\
\hline 合计 & 184 & 170 & 78 & 47 & 479 \\
\hline
\end{tabular}

以各个地震影响因素之间的相关性分析为例, 此类不 满足正态分布的定序变量, 可以利用 Kendall 相关来确定 相关性水平, 其相较常用的 Pearson 相关检验精确度较 低, 但实用性较广。使用统计软件 SPSS20.0 进行 Kendall 相关性检验, 结果如表 5 所示, 其检验概率均为 0.000 , 说明了地震影响因素之间存在显著相关。

表 5 相关系数

\begin{tabular}{|c|c|c|c|c|c|c|c|c|c|c|c|c|c|}
\hline & $d z 01$ & $d z 02$ & $d z 03$ & $d z 04$ & $d z 05$ & $d z 06$ & $d z 07$ & $d z 08$ & dz09 & $d z 10$ & $d z 11$ & $d z 12$ & $d z 13$ \\
\hline$d z 01$ & 1.00 & & & & & & & & & & & & \\
\hline$d z 02$ & $0.645^{* *}$ & 1.00 & & & & & & & & & & & \\
\hline$d z 03$ & $0.608^{* *}$ & $0.719^{* *}$ & 1.00 & & & & & & & & & & \\
\hline$d z 04$ & $0.534^{* *}$ & $0.520^{* *}$ & $0.555^{* *}$ & 1.00 & & & & & & & & & \\
\hline$d z 05$ & $0.320^{* *}$ & $0.358^{* *}$ & $0.371^{* *}$ & $0.451^{* *}$ & 1.00 & & & & & & & & \\
\hline$d z 06$ & $0.136^{* *}$ & $0.173^{* *}$ & $0.158^{* *}$ & $0.237^{* *}$ & $0.303^{* *}$ & 1.00 & & & & & & & \\
\hline$d z 07$ & $0.261^{* *}$ & $0.244^{* *}$ & $0.241^{* *}$ & $0.286^{* *}$ & $0.373^{* *}$ & $0.377^{* *}$ & 1.00 & & & & & & \\
\hline$d z 08$ & $0.260^{* *}$ & $0.287^{* *}$ & $0.307^{* *}$ & $0.393^{* *}$ & $0.473^{* *}$ & $0.218^{* *}$ & $0.301^{* *}$ & 1.00 & & & & & \\
\hline$d z 09$ & $0.484^{* *}$ & $0.464^{* * *}$ & $0.484^{* *}$ & $0.403^{* *}$ & $0.267^{* *}$ & $0.132^{* *}$ & $0.295^{* *}$ & $0.236^{* *}$ & 1.00 & & & & \\
\hline$d z 10$ & $0.306^{* *}$ & $0.326^{* * *}$ & $0.373^{* *}$ & $0.430^{* *}$ & $0.422^{* *}$ & $0.281^{* *}$ & $0.277^{* *}$ & $0.486^{* *}$ & $0.351 .^{* *}$ & 1.00 & & & \\
\hline$d z 11$ & $0.276^{* *}$ & $0.313^{* * *}$ & $0.352^{* *}$ & $0.307^{* *}$ & $0.378^{* *}$ & $0.237^{* *}$ & $0.278^{* *}$ & $0.462^{* *}$ & $0.283^{* *}$ & $0.544^{* *}$ & 1.00 & & \\
\hline$d z 12$ & $0.287^{* *}$ & $0.293^{* *}$ & $0.320^{* *}$ & $0.320^{* *}$ & $0.334^{* *}$ & $0.196^{* *}$ & $0.249^{* *}$ & $0.436^{* *}$ & $0.299^{* * *}$ & $0.504^{* *}$ & $0.745^{* *}$ & 1.00 & \\
\hline$d z 13$ & $0.451^{* *}$ & $0.424^{* * *}$ & $0.461^{* *}$ & $0.383^{* *}$ & $0.267^{* *}$ & $0.180^{* *}$ & $0.282^{* *}$ & $0.254^{* *}$ & $0.469^{* *}$ & $0.287^{* *}$ & $0.263^{* *}$ & $0.258^{* *}$ & 1.00 \\
\hline
\end{tabular}

1 ) **表示在置信度 (双侧) 为 0.01 时, 相关性是显著的

表 6 解释的总方差

\begin{tabular}{ccccccc}
\hline \multirow{2}{*}{ 成份 } & \multicolumn{3}{c}{ 初始特征值 } & 累计\% & 合计 & 旋转平方和载入 \\
\cline { 2 - 6 } & 合计 & 方差的\% & 累计\% \\
\hline 降水量 $h s_{01}$ & 7.092 & 47.280 & 47.280 & 5.100 & 33.999 & 33.999 \\
淹没范围 $h s_{02}$ & 2.265 & 15.099 & 62.379 & 2.983 & 19.885 & 53.884 \\
淹没深度 $h s_{03}$ & 1.080 & 7.198 & 69.577 & 2.354 & 15.693 & 69.577 \\
淹没时长 $h s_{04}$ & 0.694 & 4.629 & 74.207 & & & \\
最大径流量 $h s_{05}$ & 0.569 & 3.796 & 78.003 & & \\
农田分布情况 $h s_{06}$ & 0.528 & 3.521 & 81.524 & & \\
洪水房屋结构 $h s_{07}$ & 0.509 & 3.396 & 84.920 & & \\
洪水经济发达程度 $h s_{08}$ & 0.421 & 2.807 & 87.727 & & \\
洪水人口密度 $h s_{09}$ & 0.380 & 2.534 & 90.261 & & \\
洪水居民自救互救能力 $h s_{10}$ & 0.332 & 2.213 & 92.474 & & \\
洪水发生时间 $h s_{11}$ & 0.309 & 2.062 & 94.535 & & \\
洪水基础设施完备程度 $h s_{12}$ & 0.293 & 1.954 & 96.489 & & \\
洪水救援力度 $h s_{13}$ & 0.208 & 1.385 & 97.874 & & \\
洪水救援速度 $h s_{14}$ & 0.186 & 1.239 & 99.113 & & \\
洪水是否引起次生灾害 $h s_{15}$ & 0.133 & 0.887 & 100.000 & & \\
\hline
\end{tabular}

从表 5 相关系数表可以看出, 地震影响因素各个变量 存在相关性关系, 而洪水灾情影响因素各个变量之间也 存在着相关性, 相关性分析过程省略, 可以进行因子分 析。
D. 因子分析

因为灾情影响因素各个变量之间存在着相关性, 故可 用因子分析对变量进行降维, 以洪水灾情影响因素为 例, 首先通过 $\mathrm{KMO}$ 和 Bartlett 球形检验, $\mathrm{KMO}$ 值为 0.915, 表示非常适合做因子分析, Bartlett 球形检验值的 近似卡方为 4719.370 , 检验概率 sig 值为 0.000 , 因此可 以认为本案例中观测变量的相关系数矩阵与单位矩阵有 
显著差异，所以选取的指标做因子分析具有一定的合理 性。

因子分析抽取方法, 选取主成份法, 抽取基于特征值 大于 1 的主成分。为了获得更好的分析结果, 采用最大 方差法对因子进行旋转变换, 使各个主成分的载荷值相 对集中, 从而能比较容易地解释各个主因子的含义。解 释的总方差如表 6 所示, 系统预设了 15 个主成分, 但经 过几次迭代, 只有 3 个成分的初始特征值 $>1$, 且旋转后 的 3 个成分解释的累计方差量为 $69.577 \%$, 代表了待分 析变量的大部分信息。因此, 提取三个主成分是合理 的。

表 7 旋转成分所示: 公共因子 $F_{1}$ 与降水量、淹没范 围、淹没深度、淹没时长、最大径流量、洪水发生时 间、洪水是否引起次生灾害有较高的载荷, 这些都反映 了风险源的水平, 可以称公共因子 $F_{1}$ 为风险源因子; 公 共因子 $F_{2}$ 与居民自救互救能力、基础设施完备程度、救 援力度、救援速度有较高的载荷, 尤其救援力度和救援 速度比较高, 这些主要反映了当地应急救援的水平, 可 以称公共因子 $F_{2}$ 为应急能力因子; 公共因子 $F_{3}$ 与农田 分布情况、房屋结构、经济发达程度和人口密度有较高 的载荷, 这些都反映了风险承受体的脆弱性水平, 可以 称公共因子 $F_{3}$ 为脆弱性因子。地震灾情影响因素与洪水 灾情影响因素的结论相仿, 过程省略。

表 7 旋转成分矩阵

\begin{tabular}{cccc}
\hline 原始指标 & \multicolumn{3}{c}{ 公共因子 } \\
\cline { 2 - 4 } & $\boldsymbol{F}_{\mathbf{1}}$ & $\boldsymbol{F}_{\mathbf{2}}$ & $\boldsymbol{F}_{\mathbf{3}}$ \\
\hline 降水量 $h s_{01}$ & $\mathbf{0 . 7 8 7}$ & 0.232 & 0.091 \\
淹没范围 $h s_{02}$ & $\mathbf{0 . 8 6 6}$ & 0.091 & 0.167 \\
淹没深度 $h s_{03}$ & $\mathbf{0 . 8 9 4}$ & 0.120 & 0.109 \\
淹没时长 $h s_{04}$ & $\mathbf{0 . 8 6 6}$ & 0.115 & 0.143 \\
最大径流量 $h s_{05}$ & $\mathbf{0 . 7 9 1}$ & 0.173 & 0.219 \\
农田分布情况 $h s_{06}$ & 0.525 & 0.119 & $\mathbf{0 . 5 5 0}$ \\
洪水房屋结构 $h s_{07}$ & 0.213 & 0.463 & $\mathbf{0 . 5 9 5}$ \\
洪水经济发达程度 $h s_{08}$ & 0.039 & 0.171 & $\mathbf{0 . 8 2 3}$ \\
洪水人口密度 $h s_{09}$ & 0.393 & 0.162 & $\mathbf{0 . 6 2 4}$ \\
洪水居民自救互救能力 $h s_{10}$ & 0.106 & $\mathbf{0 . 7 1 6}$ & 0.407 \\
洪水发生时间 $h s_{11}$ & $\mathbf{0 . 7 7 1}$ & 0.103 & 0.302 \\
洪水基础设施完备程度 $h s_{12}$ & 0.214 & $\mathbf{0 . 6 3 0}$ & 0.482 \\
洪水救援力度 $h s_{13}$ & 0.175 & $\mathbf{0 . 8 9 5}$ & 0.093 \\
洪水救援速度 $h s_{14}$ & 0.215 & $\mathbf{0 . 8 8 6}$ & 0.093 \\
洪水是否引起次生灾害 $h s_{15}$ & $\mathbf{0 . 5 9 2}$ & 0.274 & 0.091 \\
\hline
\end{tabular}

由表 8 可得:

$F_{1}=0.186 * h s_{01}+0.204 * h s_{02}+0.220 * h s_{03}+0.207 * h s_{04}+0.17$ $0 * h s_{05}+0.046 * h s_{06}-0.070 * h s_{07}-0.135 * h s_{08}-0.005 * h s_{09}$ $0.086 * h s_{10}+0.155 * h s_{11}-0.063 * h s_{12}-0.028 * h s_{13}-$ $0.017 * h s_{14}+0.129 * h s_{15}$.

$F_{2} 、 F_{3}$ 过程同理, 略去。这里的变量, $F_{1} 、 F_{2}$ 、 $F_{3} 、 h s_{01}-h s_{15}$ 是标准正态变换后的变量。

\section{IV. 结论与讨论}

通过对三台县 479 份关于民众对于地震、洪水灾情 影响因素重要性评分的调查, 并结合个体因素进行分 析, 发现不同背景, 如不同年龄段、户籍、职业与其打 分相关性很高, 尤其是职业为学生的人, 与非学生的人 打分差异性很大, 由于所选学生小学生比例高一些, 他 们可能不太能理解了关于影响因素复杂的专业词汇, 这
表 8 成份得分系数矩阵

\begin{tabular}{cccc}
\hline 原始指标 & \multicolumn{3}{c}{ 公共因子 } \\
\cline { 2 - 4 } & $\boldsymbol{F}_{\mathbf{1}}$ & $\boldsymbol{F}_{\mathbf{2}}$ & $\boldsymbol{F}_{\mathbf{3}}$ \\
\hline 降水量 $h s_{01}$ & 0.186 & 0.040 & -0.126 \\
淹没范围 $h s_{02}$ & 0.204 & -0.059 & -0.046 \\
淹没深度 $h s_{03}$ & 0.220 & -0.030 & -0.100 \\
淹没时长 $h s_{04}$ & 0.207 & -0.040 & -0.069 \\
最大径流量 $h s_{05}$ & 0.170 & -0.026 & -0.018 \\
农田分布情况 $h s_{06}$ & 0.046 & -0.126 & 0.279 \\
洪水房屋结构 $h s_{07}$ & -0.070 & 0.058 & 0.269 \\
洪水经济发达程度 $h s_{08}$ & -0.135 & -0.141 & 0.542 \\
洪水人口密度 $h s_{09}$ & -0.005 & -0.116 & 0.342 \\
洪水居民自救互救能力 $h s_{10}$ & -0.086 & 0.247 & 0.080 \\
洪水发生时间 $h s_{11}$ & 0.155 & -0.083 & 0.065 \\
洪水基础设施完备程度 $h s_{12}$ & -0.063 & 0.175 & 0.140 \\
洪水救援力度 $h s_{13}$ & -0.028 & 0.420 & -0.207 \\
洪水救援速度 $h s_{14}$ & -0.017 & 0.413 & -0.211 \\
洪水是否引起次生灾害 $h s_{15}$ & 0.129 & 0.074 & -0.106 \\
\hline
\end{tabular}

一点在线下论坛中也有发现, 所以在今后的调查中需要 对复杂的专业词汇做出书面或口头解释。

通过影响因素指标间的因子分析, 再结合指标的整体 分析, 可以发现三台县民众比较看重自然属性的风险源 水平和注重政府的应急能力, 最后是风险承受体的脆弱 性水平。在与民众接触过程中, 发现他们对于灾害的态 度是“小灾不用躲, 大灾躲不过”, 比较消极, 当地政府 应该普及灾害的基础知识、自救知识, 让民众不要太怵 灾害。

也可以利用因子分析总结出的公共因子公式来为相对 暴露度公式中的权重计算提供一个思路。 是:

风险承受体与风险源关系决定的 $B$ 相对 $A$ 的暴露度

$$
E_{A(\alpha)}(B)=\sum_{i=1}^{n} w_{\alpha_{i}} E_{A, \alpha_{i}}(B)
$$

其中, $\alpha_{i}, i=1,2, \ldots, n$ 是关系参数;

$E_{A, \alpha_{i}}(B), i=1,2, \cdots, n$ 是 $B$ 相对于 $A$ 的暴露度;

$w_{\alpha_{i}}, i=1,2, \ldots, n$ 是关系参数对灾情的影响权重, 且 $\sum_{i=1}^{n} w_{\alpha_{i}}=1$ 。

举例说明, 假设 $E_{A(\alpha)}(B)$ 指的是风险承受体与风险源 (洪水) 关系决定的相对暴露度。故可以借助表 8 成份 得分系数矩阵中公共因子 $F 1$ 中的成份得分系数来衡量 相对暴露度的权重, 即关系参数 $\alpha_{i}, i=1,2, \ldots, 15$ 分别为 降水量、淹没范围、淹没深度、淹没时长、最大径流 量、农田分布情况、洪水房屋结构、洪水经济发达程 度、洪水人口密度、洪水居民自救互救能力、洪水发生 时间、洪水基础设施完备程度、洪水救援力度、洪水救 援速度、洪水是否引起次生灾害; 又由于表 8 中 $\mathrm{F} 1$ 得 分系数总和为 0.913 , 需要将成份得分系数进行归一 化, 所以就得到了上述关系参数对灾情的影响权重为 $w_{\alpha_{i}}, i=1,2, \ldots, 15$, 分别为 $0.204 、 0.223 、 0.241$ 、 $0.227 、 0.186 、 0.050 、-0.077 、-0.148 、-0.005 、-0.094$ 、 $0.170 、-0.069 、-0.031 、-0.019 、 0.141$ 。通过此案例, 期 望为相对暴露度公式中权重的计算提供一个思路。 


\section{致谢}

本项目受国家重点研发计划课题 “重大自然灾害多层 级精准救助关键技术研究” (编号：2017YFC1502902)

和国家自然科学基金项目“独立多灾种年度财产损失综合 风险的信息扩散合成范式研究”（编号：41671502）联合 资助。

\section{参考文献}

[1] 苏桂武,马宗晋,等“汶川地震灾区民众认知与响应地震灾害的特 点及其减灾宣教意义一—以四川省德阳市为例” 地震地 质,vol.30,issue 4, pp.877-894,2008.

[2] 谢晓非,李洁,于清源.“怎样会让我们感觉更危险——险沟通渠 道分析,”心理学报,vol 4 issue 3,pp.456-465.2008.

[3] 黄崇福, “自然灾害风险相对暴露度的研究, ”第六届中国西部风 险分析与风险管理学术研讨会论文集（2019 年 11 月 16-17日, 中国，昆明）.巴黎：亚特兰蒂斯出版社，pp.1-5, 2019.
[4] Shannon McCurdy,Annette Molinaro,Lior Pachter. "Factor analysis for survival time prediction with informative censoring and diverse covariates,"Statistics in Medicine, vol 38. 2019.

[5] Mansi Tripathi, Sunil Kumar Singal, "Allocation of weights using factor analysis for development of a novel water quality index" Ecotoxicology and Environmental Safety,vol.183, pp.109510, 2019.

[6] 李小胜,陈珍珍. “如何正确应用 SPSS 软件做主成分分析, ” 统计研 究,vol 27, issue 8,pp.105-108, 2010.

[7] 曹烨,邹振东,等. “基于因子分析的深圳市水资源与能源安全形势 分析,”北京大学学报 (自然科学版), vol 54, issue 6, pp.13461350,2018 .

[8] 苏筠,谢静晗,等.“汶川地震对北京公众灾害认知的影响,”地震研 究, vol 34, issue 03, pp. 378-383+404,2011.

[9] 杨威,杨霆. “体育社会学研究中定类、定序变量的回归分析, ” 体育 学刊, vol 5, pp. 22-24, 2004

[10] Atanu Biswas, Eunsik Park, "Measures of association for nominal categorical variables"Journal of the Korean Statistical Society,vol 38, issue 3, pp.247-258. 2009. 